- Acute dental pain often leads to attendances at accident and emergency (A\&tE) departments outside normal working hours.

- Three cases of unintentional overdoses of simple analgesia secondary to acute dental pain, with potentially serious consequences, are reported here.

- An accurate drug history from patients with acute dental pain is vital; every effort should be made to educate patients about simple analgesia and access to emergency dental care, especially outside normal working hours.

\title{
Unintentional overdose of analgesia secondary to acute dental pain
}

\author{
M. D. Dodd ${ }^{1}$ and C. A. Graham ${ }^{2}$
}

\begin{abstract}
Three cases of unintentional overdose with simple analgesics are presented. Over a two month period, these patients presented to the accident and emergency (A\&E) department with acute dental pain, outside normal working hours, having been unable to access emergency dental care. In one case the patient's reason for attendance was to obtain further supplies of analgesics. The patients required admission for assessment of the severity of the overdose in addition to advice about appropriate use of analgesics and advice on access to dental care. None of the patients required treatment for the overdose. These cases serve as a timely reminder of the importance of taking an accurate drug history in emergency situations. They also raise issues of patient education for self medication and access to emergency dental services outside normal working hours.
\end{abstract}

While patients with dental pain comprise a small proportion of A\&tE attendances, A\&tE staff will spend a considerable time seeing patients with toothache, often at night or at weekends when staffing levels may be less. Patients with dental pain will usually be triaged into less urgent categories and may have a long wait in order to be given analgesics or antibiotics which may be inappropriate or ineffective. Many patients will however have taken over-the-counter analgesic medication prior to attendance.

There is one published case of liver failure following paracetamol overdose for pericoronitis. ${ }^{1}$ We present three cases of unintentional overdoses of analgesia in patients suffering from acute dental pain.

${ }^{1}$ Specialist Registrar in Oral \& Maxillofacial Surgery, University Hospital, Aintree, Liverpool ${ }^{2}$ Specialist Registrar in Accident \&t Emergency Medicine, Southern General Hospital, Glasgow G51 4TF

Correspondence to:Mr C A Graham, Specialist Registrar in Accident \&t Emergency Medicine, Southern General Hospital, 1345 Govan Road, Glasgow G51 4TF

Email: colingrahamlt@ukonline.co.uk

\section{Refereed Paper}

Received 06.08.01; Accepted 22.02.02

๑ British Dental Journal 2002; 193: 211-212
Given the number of cases noted here over a two month period in an A\&tE department which registered 44,602 attendances in $1999,{ }^{2}$ there are probably more 'near misses' than has previously been thought.

\section{CASE REPORT 1}

A 17-year-old woman attended ActE at 2000h one Saturday evening having taken eight 300mg aspirin tablets and one $600 \mathrm{mg}$ ibuprofen tablet over a four hour period for toothache. She denied taking any paracetamol, opioid or compound analgesics. The preceding day, she had developed intermittent symptoms of acute pulpitis, arising from an upper left molar tooth. The pain had become constant and severe on the day of presentation. She had attended an NHS dentist in the preceding six months, but had made no attempt to contact the dental surgery as she thought no one would be there. She was brought to A\&tE by her boyfriend who was concerned that she could have taken harmful amounts of aspirin.

On examination the patient was distressed with toothache despite the analgesia. She was haemodynamically normal and apyrexial. She had no epigastric pain or tinnitus (symptoms of salicylate toxicity). She had no facial swelling and the upper left molar tooth was heavily restored but not tender to percussion. Despite the low probability of a significant overdose, she was admitted for measurement of plasma salicylate levels which were well below the treatment level. She was discharged later that night with compound analgesia (paracetamol and codeine) and instructions on its safe dosage. She was referred to the Glasgow Dental Hospital emergency clinic the following morning as it was not possible to contact her own dentist's out of hours service.

\section{CASE REPORT 2}

A 28-year-old woman self presented to the ActE department at one Monday evening having taken $11 \mathrm{~g}$ of paracetamol (22 tablets) over a 33 hour period for toothache. She presented four hours after the last dose. She was small in stature and therefore considered to be at high risk of paracetamol toxicity.

Given the delay in presentation and the fact this was a staggered overdose, expec- 
tant treatment with intravenous $\mathrm{N}$-acetylcysteine was started. Serum paracetamol levels, coagulation studies and liver function tests were checked and were normal. She was discharged with advice about safe analgesic self medication and was advised to seek an appointment with her dentist in the morning.

\section{CASE REPORT 3}

A 28-year-old male attended ActE in the early hours one Saturday morning requesting analgesia for toothache which had started 36 hours earlier. He did not volunteer what medication he had been taking and the attending A\&tE doctor did not elicit any drug history from the patient. He was prescribed ibuprofen tablets, $600 \mathrm{mg}$ three times daily and advised to seek a dental opinion.

He returned 11 hours later requesting further analgesia. Further questioning discovered that he had taken $2.4 \mathrm{~g}$ of oral ibuprofen since the previous attendance in addition to $7 \mathrm{~g}$ (14 tablets) of paracetamol over seven hours the preceding evening. He described symptoms of irreversible pulpitis arising in the upper right first molar. Although he was registered with a dental practitioner, he had made no attempt to contact the surgery as it was now the weekend. Despite the toothache starting during the week, he had not made contact with his dentist as he was hopeful that the pain would settle.

Attempts were made to contact his emergency dental service and answerphone messages were left but not replied to. Arrangements were made for him to attend the emergency dental clinic at Glasgow Dental Hospital the following day. He was admitted for serum paracetamol levels, which were normal. Coagulation studies and liver function tests were also normal and he was discharged later that evening.

He reattended again just before midnight requesting further analgesia. Repeat examination revealed tenderness to percussion and tenderness in the buccal sulcus suggestive of abscess formation. Bupivacaine was infiltrated to provide overnight analgesia and he was given Amoxicillin $500 \mathrm{mg}$ three times daily. He was given cocodamol and diclofenac with strict verbal and written instructions regarding their safe dosage and the risks of repeat overdosing. The attending doctor felt he understood these instructions clearly enough to allow the paracetamol containing analgesic to be safely dispensed.

\section{DISCUSSION}

Two of the three patients in this paper were unaware of the risks imposed by their excessive self administered analgesia. A previous study has shown a similar lack of awareness of the potential risks of excessive self medication for odontalgia in the general public. ${ }^{3}$ That study showed that most cases occur with paracetamol or compound analgesics and that most patients who excessively self medicate are smokers. Both psychological and pharmacological explanations were offered to explain this. Our series shows a similar but less marked pattern.

Despite an abundance of literature on analgesia for dental pain, there is little published work on the use or abuse of over-the-counter medications. There are two published cases of overdose requiring therapeutic intervention; one resulting in hepatic failure after overdose of paracetamol for pericoronitis ${ }^{1}$ and the second when a patient took a non-steroidal anti-inflammatory drug which is normally prescribed by vets to horses! ${ }^{4}$ This series suggests that accidental overdose may be more common than previously thought.

Serum paracetamol levels are useful in determining treatment requirements when the time of the overdose is known with certainty. When an overdose is staggered, serum paracetamol tests are impossible to interpret and the clinician has to rely on surrogate markers of liver damage, such as liver function tests and the coagulation profile.

The presence of normal liver function tests and a normal coagulation screen virtually excludes significant hepatotoxicity after minor paracetamol overdose.
Although no patient in this series suffered serious toxicity, these incidents highlight the importance of patient education and accurate drug histories however trivial or inappropriate the attendance is felt to be.

These cases also highlight the issue of out of hours emergency dental care. It suggests that even patients who are registered with a dentist may not know how to access emergency dental services. It then falls to other health service providers to meet these needs (for example, emergency general medical practitioner services, ACtE departments, walk-in centres, pharmacies and dental hospitals). This can be time consuming and distressing for the patient and they may receive sub-optimal care from staff without specific dental training or skills.

We are unaware of any published work on attendances to general ActE departments with toothache in the United Kingdom. It is our experience that many inappropriate visits to ActE could be averted with simple advice on appropriate overthe-counter medication, an awareness of emergency dental service provision and advice on how to access them. This may also lessen the risks of significant accidental overdose of commonly available analgesics. Proactive patient education and provision of written and verbal information may be an important step in improving access to emergency dental services.

The authors would like to thank Mr MW G Gordon, Dr T R J Parke and Dr P T Munro, Consultants in Accident \& Emergency Medicine at the Southern General Hospital in Glasgow, for permission to report these cases. We particularly thank Mr Gordon for details of Case 2.

1 Sivalognathan $\mathrm{K}$, Johnson $\mathrm{PA}$, Bray G P, Williams $\mathrm{R}$. Pericoronitis and accidental paracetamol overdose, a cautionary tale. Br Dent J 1993; 174: 69-71.

2 British Association for Accident \& Emergency Medicine Directory 2001/2002. BOE Publishing. London. 2001. p173.

3 Preshaw P M, Meechan J G, Dodd M D. Self medication for the control of dental pain: what are our patients taking? Dent Update 1994; 21: 299-304.

4 Newton TA, Rose SR. Poisoning with equine phenylbutazone in a racetrack worker. Ann Emerg Med 1991; 20: 204-207. 